\title{
Direct Measurement of the Transverse Modulus of Carbon Fibres
}

\author{
S. Wong ${ }^{1}$ (D) A. P. Pierlot ${ }^{1}$ - A. M. Abbott ${ }^{1}$ - J. A. Schutz ${ }^{1}$
}

Received: 17 March 2021 / Accepted: 13 January 2022 / Published online: 27 January 2022

(c) Crown 2022

\begin{abstract}
Background The transverse compressive properties are integral to fully utilizing the high performing mechanical properties of carbon fibres (CF). Direct measurements of transverse properties of CFs are difficult.

Objective A system that directly measures transverse compressive modulus $\left(E_{T}\right)$ by utilizing interference speckle patterns to measure sub-pixel resolutions is presented.

Methods The compression data from an isotropic glass fibre was fitted using an elastic contact mechanics model. An initial section was observed, which was not readily fitted using the model and was systematically removed, resulting in an $E_{T}$ of 82 $\mathrm{GPa}$, comparable to the reported longitudinal tensile modulus $\left(E_{L}\right)$ of $86 \mathrm{GPa}$.

Results The response of Ag wire to cyclic compression was measured, with the behaviour consistent with a material undergoing typical cyclic stress-strain into a stable hysteretic loop. Several CFs were compressed and an inversely proportional relationship between $E_{T}$ and $E_{L}$ was observed. Transverse compressive moduli of $8.2(0.8), 6.5(0.7), 4.3(0.3)$, and $2.1(0.4)$ were obtained for Toray T300 and Mitsubishi Pyrofil HS40, Pyrofil TR50S, and Dialead K13312, respectively (numbers in parenthesis are standard deviations).
\end{abstract}

Conclusion Hysteresis was observed for some of the polyacrylonitrile (PAN)-based CFs and a method for ensuring an accurate fitting of $\mathrm{CFs}$, including hysteretic load/unload curves, was proposed.

Keywords Carbon fibre $\cdot$ Mechanical properties · Transverse compression $\cdot$ Hysteresis

\section{Introduction}

Carbon fibre (CF) is an important reinforcing material in composites due to a combination of its high performing mechanical properties and relatively low density. Their high strength and high modulus under tensile stress have made carbon fibres ideal reinforcements in composites for the aerospace and energy generation industries [1], while the high damage tolerance of carbon fibre reinforced ceramics have great potential for space-based applications [2]. Thus, the tensile strength and modulus, and the microstructures that give rise to these desirable properties, have been the focus of many studies [3-5].

The compressive properties of CFs and their dependence on the fibre's microstructure are not as well understood. The high-performing tensile properties arise from the high microstructural alignment of hexagonal carbon sheets along the fibre

S. Wong

Sherman.wong@csiro.au

1 CSIRO Manufacturing, Waurn Ponds, Victoria 3216, Australia axis with weaker forces between sheets in lateral direction. This anisotropy results in significant difference between the mechanical properties in the longitudinal direction (along the fibre) and the transverse direction (perpendicular to the fibre axis) [6-8] as well as between their tensile and compressive properties $[9,10]$. In practical applications, most carbon fibre reinforced materials fail not under tensile stress but under compression due to a significantly lower compressive strength. For example, Oya et al. reports that a reduction in strength of 50 $-70 \%$ is observed when the single fibre is exposed to compressive stresses rather than tensile [9], which in turn negatively impacts the strength of composites containing these CFs [10]. While studies have investigated the compressive properties of CF in a composite $[11,12]$, it is necessary to study single fibres to fully understand its mechanical properties and their relationship to the fibre's microstructure. Indeed, such values are critical for the finite element analysis in a bottoms-up multiscale modelling approach [13]. However, even recent modelling studies $[14,15]$ have had to rely on $\mathrm{E}_{\mathrm{T}}$ values obtained indirectly via Raman microscopy and nanoindentation [16] or estimated from other mechanical properties [17]. 
Previous single fibre studies have measured longitudinal strength by observing breaks in the stress/strain curve of a single fibre under tensile loading [18-20]. However, direct measurement of compressive moduli (in both transverse and longitudinal directions) were not as readily measured. This has been suggested to be due to the large experimental error resulting from measuring the machine compliance at low force near the detector resolution limit [9], with this issue amplified for samples, such as $\mathrm{CF}$, that have a low range of initial elastic deformation [21]. Different methods have been used to overcome this limitation. Some studies to determine the compressive modulus have been performed using indentation, with the modulus calculated using the standard Oliver and Pharr model (indentation modulus) $[16,22]$ or by first calculating the stiffness coefficients $[11,23]$. However, these studies noted a significant difference between the indentation modulus and the transverse modulus calculated via the stiffness coefficients, with this difference reported to vary depending on the microstructure of the CF [23]. Other studies have compressed the fibre between two flat surfaces and measured the displacement during compression using a capacitor $[24,25]$ or linear differential transformer [7] external to the region where fibre deformation is occurring. For these systems, the machine compliance of the equipment between the deformation region and the displacement detector must be estimated and may result in an error in the final result.

Here, a compression device was developed that measures the elastic modulus of a single $\mathrm{CF}$ along the transverse direction by compressing the fibre between two diamond tips while directly observing the displacement using low coherence, interferometric speckle tracking software. The innovation of this device is that it overcomes the compliance issues by directly measuring tip displacement during compression, using tips with substantially higher modulus than the compressed fibre. Several isotropic fibres were studied to validate the accuracy of the system. Three different response regimes were identified in these standard samples and a novel method for quantitatively isolating the desired regime in which the compressive transverse modulus $\left(E_{T}\right)$ can be derived using a Hertzian contact mechanics model is presented. Several CFs were tested using this setup, including a $\mathrm{CF}$ that displayed hysteretic behaviour. The hysteretic behaviour did not change the fitted $\mathrm{E}_{\mathrm{T}}$ and a method for differentiating hysteretic curves and plastic curves is presented.

\section{Materials and Method}

\section{Data Fitting}

Various theoretical models based on Hertzian contact mechanics have been developed to describe the elastic deformation of a cylinder (e.g. circular fibre) under transverse compression. A recent comparison study of the different models [6] on $20-25 \mathrm{~mm}$ diameter poly(methyl methacrylate) rods showed that the experimental data was most accurately fitted by the model developed by Morris et al. [26] which defines the displacement $(U)$ as:

$U=\frac{4 F}{\pi}\left(\frac{1}{E_{T}}-\frac{v^{2}}{E_{L}}\right)\left(\sinh ^{-1}\left(\frac{R}{b}\right)\right)$

where $F$ is the force per unit length $\left(\mathrm{N} \cdot \mathrm{m}^{-1}\right), E_{T}$ is the compressive transverse modulus $(\mathrm{Pa}), E_{L}$ is the compressive longitudinal modulus $(\mathrm{Pa}), \nu$ is Poisson's ratio, $R$ is the radius of the fibre (m), and $b$ is the contact segment width between the fibre and the tip, which is given by:

$b=\sqrt{\frac{4 F R}{\pi}\left(\frac{1}{E_{T}}-\frac{v^{2}}{E_{L}}\right)}$

For functional fitting of transverse compression measurements, it is necessary to rewrite Eq. (1) in terms of fit parameter $k$ and derive $E_{T}$ from the fit parameter:

$k=\left(\frac{1}{E_{T}}-\frac{v^{2}}{E_{L}}\right)$

$E_{T}=\frac{1}{\left(k+\frac{v^{2}}{E_{L}}\right)}$

However, for $\mathrm{CF}$, due to its anisotropic nature, one can assume $E_{T}<<E_{L}$ making the impact of the $\frac{v^{2}}{E_{L}}$ term negligible, and simplifying the equations when applied to CFs as:

$U=\frac{4 F}{\pi}\left(\frac{1}{E_{T}}\right)\left(\sinh ^{-1}\left(\frac{R}{b}\right)\right)$

Thus, by fitting the load/displacement curve using Eq. (1) for isotropic materials and Eq. (5) for CFs, $E_{T}$ can be derived directly.

\section{Materials}

The CFs used in this study are T300 from Toray Industries and HS40 Pyrofil, TR50S Pyrofil, and K13312 Dialead from Mitsubishi Chemical Functional Products. To measure only the CF component of the studied fibres, the CFs were desized via a process of soaking fibre bundles sequentially in dimethylformamide $\left(50{ }^{\circ} \mathrm{C}, 10 \mathrm{~h}\right)$, acetone (room temperature (RT), $3 \mathrm{~h}$ ), ethanol (RT, $3 \mathrm{~h}$ ), then rinsing with cold water before drying $\left(100^{\circ} \mathrm{C}, 3 \mathrm{~h}\right)$. The glass filament used in this study is SE1200 Advantex from Owens Corning and the soft fine silver $(\mathrm{Ag})$ wire is $99.99 \%$ purity from Eurowire Limited (Great Dunmow, Essex, U.K.). Both filaments were unsized and were used as received. 


\section{Compression}

Figure 1 presents a schematic of the transverse compression device. The fibres were compressed between two flat-ended diamond tips with circular flat surfaces $100 \mu \mathrm{m}$ in diameter (Bruker Corporation) and an RMS surface roughness of $1.5 \mathrm{~nm}$ according to the manufacturer. The bottom tip is attached to a piezo driven stage (P-621ZCD, Physik Instrumente) on a manual $x-y-z$ stage that was used for aligning the tips and driving the compressive strain. The top tip is attached to a bilateral load cell with maximum load of $1.1 \mathrm{~N}$ (LRF400-FSH04037, Futek Inc.) attached to a separate translation stage. The fibre was aligned between the tips using a pair of video cameras that were oriented perpendicular to each other. One of these cameras also acted as strain sensor as it was used alongside optical speckle tracking software (iMetrum-Version 3.1) to measure the tip displacement. White light, low coherence interference speckle patterns on the tips were used as targets. The accuracy of the video gauge is dependent on the optical reflection of the targets, with the targets set on the highly speckled tungsten mounts of the tips (as seen in Fig. 1c) giving an accuracy of typically $9 \mathrm{~nm}$. The tilt between the two flat surfaces is compensated by the lateral yield of the load cell. Filaments were suspended between the centres of the tips and the bottom tip

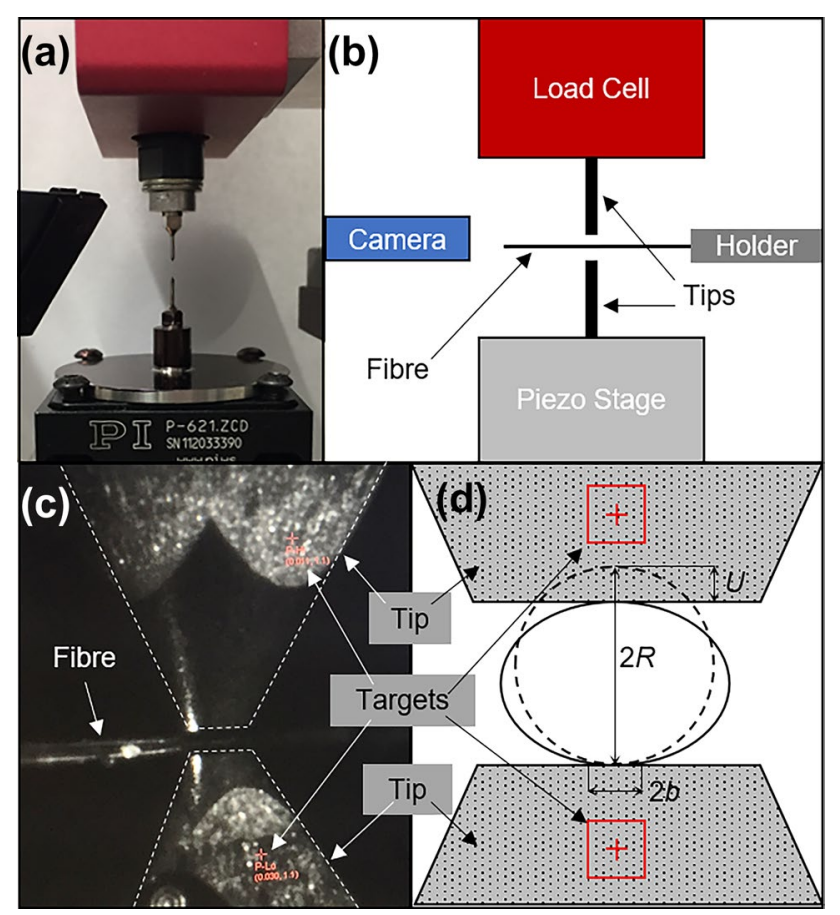

Fig. 1 (a) Photo and (b) schematic of the experimental setup. (c) Screenshot and (d) schematic of a fibre and displacement measurement targets during compression. The tips are outlined (dotted white line) for clarity. The radius $R$, contact segment width $b$, and displacement $U$ are also shown was driven towards the upper tip by the piezo drive (E-753, Physik Instrumente) with a $0.1 \mu \mathrm{m}$ step-size.

Compression of up to $700 \mathrm{mN}$, comprised of a loading segment at a rate of $0.5 \mu \mathrm{m} . \mathrm{s}^{-1}$, a hold segment of $20-40 \mathrm{~s}$, and an unloading segment at the same rate, was performed at different positions along the fibre. Repeated cyclic compression at a single position was also performed, with the tip being fully disengaged between each compression cycle. At least 10 compressions at different positions were performed for each fibre type.

\section{Sample Preparation}

For transverse compression, the fibres are mounted on a frame, secured on both ends, with a window width of $25 \mathrm{~mm}$. The fibre is freestanding and is moved between the tips using $\mathrm{X}-\mathrm{Y}$ manipulators.

As the $R$ and $E_{L}$ are required for the fitting process, Favimat + Robot 2 single fibre tester from Textechno H. Stein was used to derive $R$ and $E_{L}$ for the glass fibre and $E_{L}$ values for the CFs. A Hitachi $4000+$ SEM was used to measure the $R$ for the Ag wire and the CFs. Figure 2 presents the SEM images of the 4 CFs. The HS40 and K13312 CFs appear to have uniformly circular cross-sections. Conversely, not all T300 and TR50S CF cross-sections were completely circular, with eccentricities of up to 0.70 and 0.53 , respectively. While the Hertzian contact mechanics model assumes a circular cross-section, previous studies have fitted T300 $\mathrm{CF}[7,24]$ as well as other CFs of greater eccentricity (e.g. M60JB CF, $e=0.73$ [24]) using such models. In those studies, the fitted $\mathrm{E}_{\mathrm{T}}$ for these CFs with eccentricity up to 0.73 followed the same trends as their more circular counterparts, suggesting that any offset introduced by fitting a slightly non-circular cross-section for T300 $(e=0.70)$ and TR50S $(e=0.53) \mathrm{CF}$ is not significant. For the non-circular CFs, the used $R$ is the radius of a circle with the same area as the non-circular cross-section. The $E_{L}$ for the $\mathrm{Ag}$ wire was provided by the manufacturers. As the glass fibre and $\mathrm{Ag}$ wire are isotropic, $E_{L}$ is expected to be the same under both tensile and compressive stress within the elastic regime. The measured values as well as the Poisson ratios supplied by the manufacturers can be found in Table 1, where numbers given in parentheses are standard deviations.

\section{Results}

\section{Compression of Glass Fibre}

Figure 3(a) shows a load/displacement curve resulting from the compression of SE1200 glass fibre that has been fitted using Eq. 1. Above $\sim 200 \mathrm{mN}$ the data is well-fit by the 
Fig. 2 SEM images of the fibre cross-sections cut using sharp blade for (a) HS40, (b) K13312, (c) TR50S, and (d) T300 CF. Examples of slightly non-circular cross-sections in (c) and (d) are highlighted
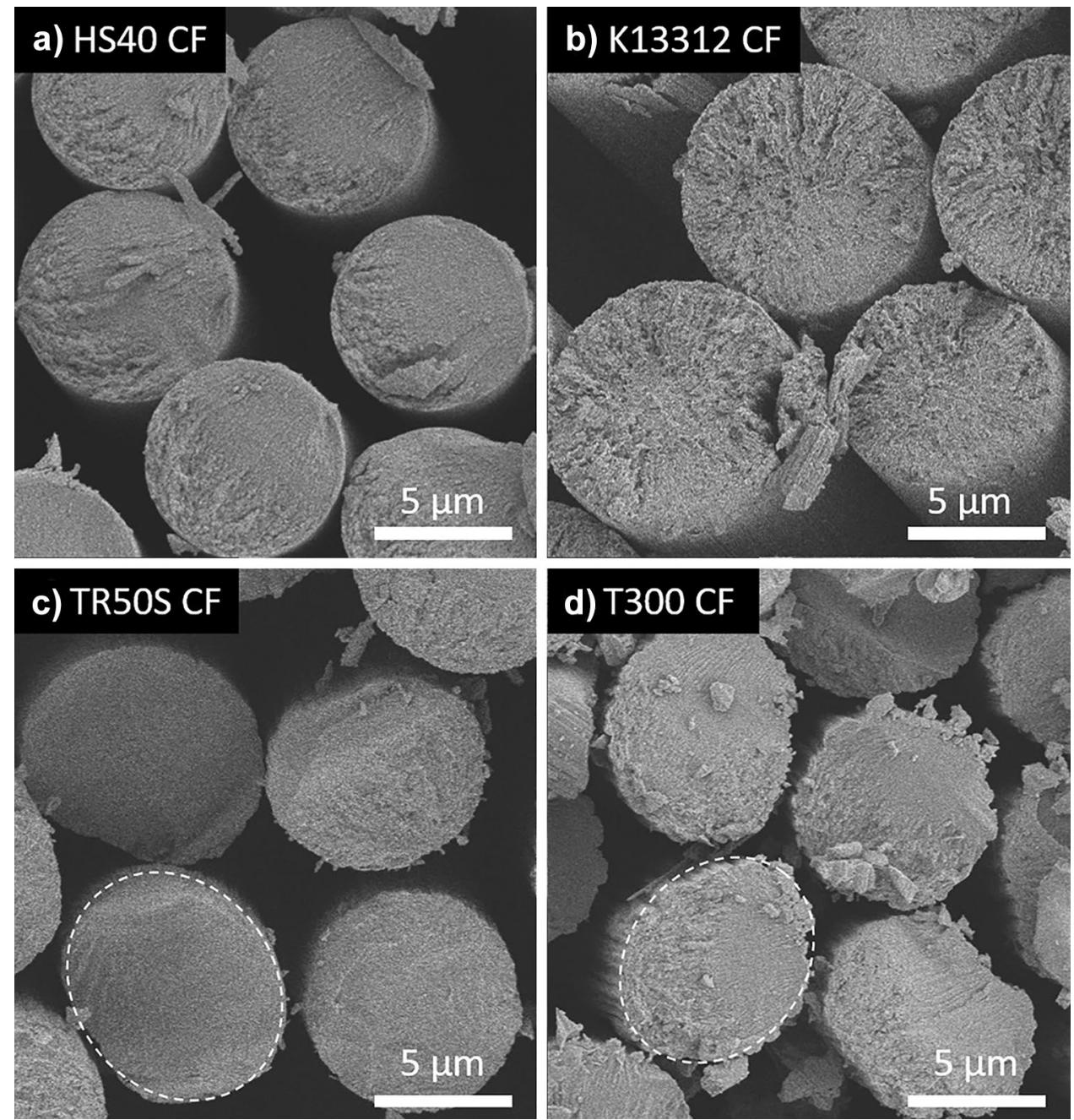

model, with $E_{T}=82(9) \mathrm{GPa}$. This value is consistent for an isotropic material with $E_{L}=86(2) \mathrm{GPa}$ as measured using the Favimat and $E_{L}=81 \mathrm{GPa}$ as specified by the manufacturer. However, there is also a section at lower loads that is not well-fitted by the model developed by Morris et al. [26] (henceforth referred to as 'initial section'). Such a section has previously been observed, and it was reported by Naito et al. that it exists due to an increase in the contact zone

Table 1 The radius, longitudinal modulus, and Poisson's ratio of the fibres tested in this study. The values with no standard deviation (in parenthesis) provided are those supplied by the manufacturers

\begin{tabular}{llll}
\hline Fibre & Diameter $(\mu \mathrm{m})$ & $\begin{array}{l}\text { Longitudinal } \\
\text { Modulus }(\mathrm{GPa})\end{array}$ & Poisson's Ratio \\
\hline SE1200 Glass & $15.2(0.6)$ & $86.3(2.3)$ & 0.22 \\
Ag & $15.1(0.6)$ & 69 & 0.38 \\
T300 & $7.0(0.2)$ & $201(4)$ & 0.27 \\
HS40 & $5.2(0.2)$ & $363(16)$ & 0.27 \\
TR50S & $6.9(0.3)$ & $203(4)$ & 0.27 \\
K13312 & $9.0(0.7)$ & $357(24)$ & 0.27 \\
\hline
\end{tabular}

within this loading range as fibres are not perfectly circular and their surfaces are not perfectly smooth [24]. It is interesting to note that, due to the direct observation utilised by this compression device, it is possible to observe slight realignment of the fibre to lie perfectly flat against the tips upon initial contact. In our experiments, the extent of this initial section increased when the fibre was misaligned during mounting (not shown). Thus, we further suggest that the movement of the fibre to orient itself parallel to the tips to maximize contact area also contributes to this initial section that was observed both here and by Naito et al. The impact of the initial section was investigated to validate its exclusion from the fitted data.

The approach to eliminating the 'initial section' is illustrated in Fig. 3(b), which plots the change in $\mathrm{E}_{\mathrm{T}}$ as the fitted load range was changed to $F=F_{\text {min }}-700 \mathrm{mN}$ (i.e. the data point at $F_{\min }=150 \mathrm{mN}$ is the $\mathrm{E}_{\mathrm{T}}$ when the data from $F=150-700 \mathrm{mN}$ was fitted), normalized to the chosen $E_{T}$. Two distinct regions can be observed. In the first, the modulus increases as more data at low displacement is excluded. In the second, the modulus remains constant, independent 


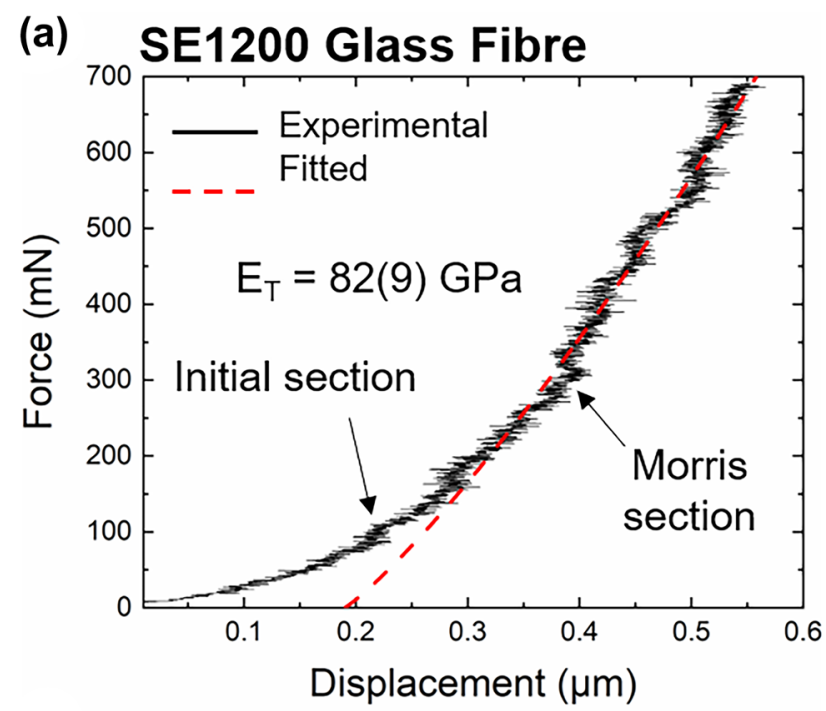

(b)

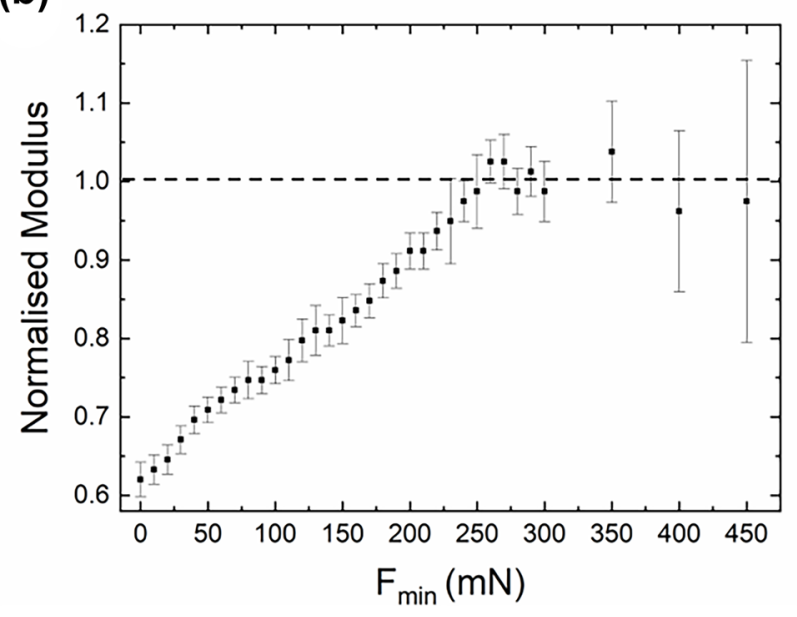

Fig. 3 (a) A typical load/displacement curve from SE1200 glass fibre fitted using the model developed by Morris et al. [26]. (b) The change in transverse modulus as the initial section (that is not well-fitted by the model) is progressively excluded from the section that is wellfitted by the model. All moduli presented here have been normalized to the chosen modulus of their respective curves. Numbers given in parenthesis are standard deviations and the error bars represent $95 \%$ confidence intervals

of the fitted range. Unsurprisingly, the confidence interval increases with increasing $F_{\min }$, as number of data points in the fitted region becomes smaller (i.e. more data is excluded from the fit). We propose that the former represents cases where both the initial section and the section that can be well-fitted by the model (Eq. 1) proposed by Morris et al. [26] (henceforth referred to as 'Morris section') are included in the fit, while the latter represents cases where only the Morris section (e.g. F $>250 \mathrm{mN}$ for the loading curves used in Fig. 3b) is included. As this method can be readily applied to all fibres, all subsequent moduli presented in this work are derived from fits that exclude the low displacement data using this method whilst recognizing that it is important to retain as much of the Morris section as possible.

At higher loads, plastic deformation of the sample is expected. As the Morris model is only accurate within the elastic regime, the section where plasticity is present (henceforth referred to as 'plastic section') must also be excluded when calculating $E_{T}$. No plastic section was observed during compression of the glass fibre up to the maximum load achieved in this study $(700 \mathrm{mN})$. To explore the impact of the plastic section, a more ductile material was compressed. Figure 4(a) shows load/displacement curves from cyclic compression of $\mathrm{Ag}$ wire. The first cycle displays clear plasticity, with a non-zero residual depth as can be seen in the inset SEM image. There appear to be two distinct sections in the loading curve, from $0-20 \mathrm{mN}$ and from $20-60$ $\mathrm{mN}$. Fitting of the curve below $20 \mathrm{mN}$ using the method presented in Fig. 3(b) resulted in an increasing modulus, indicating this is the initial section. However, attempting to fit the section above $20 \mathrm{mN}$ using the Morris model (Eq. 1) results in a derived $E_{T}=\sim 2 \mathrm{GPa}$, which is not consistent with a $E_{L}=69 \mathrm{GPa}$. This section of the loading curve is most likely dominated by the plastic deformation of the sample. As expected for a highly ductile metal, plasticity occurs at unmeasurably low loads at less than $0.2 \%$ strain [27] and the pure Morris section becomes negligible.

Subsequent cycles at the same maximum load result in a hysteretic behaviour, with curves similar in shape to the unloading curve of the first cycle. When the maximum load is increased (cycle 4 and 7), the same plasticity into stable hysteresis behaviour is observed at a higher displacement. This behaviour is comparable to that of a material undergoing cyclic stress strain (CSS) [28] under zero-to-tension uniaxial loading [29]. Here the sample experiences compression rather than tension, but the response has not been reported to be dependent on the direction of the stress. Some materials undergoing CSS are 'Masing materials', where the hysteresis loops (once offset for displacement) will coincide [30]. Critically, for a Masing material, $E_{T}$ can be derived from the loading curve of any hysteretic curve. To determine if the Ag wire is a Masing material, $E_{T}$ was derived for the hysteretic curves at a displacement of $\sim 1.2 \mu \mathrm{m}\left(E_{T}=\sim 18\right.$ $\mathrm{GPa}), 1.8 \mu \mathrm{m}\left(E_{T}=\sim 37 \mathrm{GPa}\right)$, and $\sim 2.4 \mu \mathrm{m}\left(E_{T}=\sim 57\right.$ $\mathrm{GPa})$. This indicates that the $E_{T}$ of the $\mathrm{Ag}$ wire cannot be derived in this manner. Further, a highly likely explanation for the increasing trend in $E_{T}$ is that the wire is becoming flatter with increasing plastic deformation. This suggests that the subsequent hysteretic curves after the initial onset of plasticity cannot be utilized to derive $E_{T}$ using the Morris model for any fibre. Thus, an accurate $E_{T}$ could not be calculated for the Ag wire.

In contrast, Fig. 4(b) shows cyclic compression on HS40 CF where the curves show hysteretic behaviour without an initial onset of plasticity. To explore the possibility of 

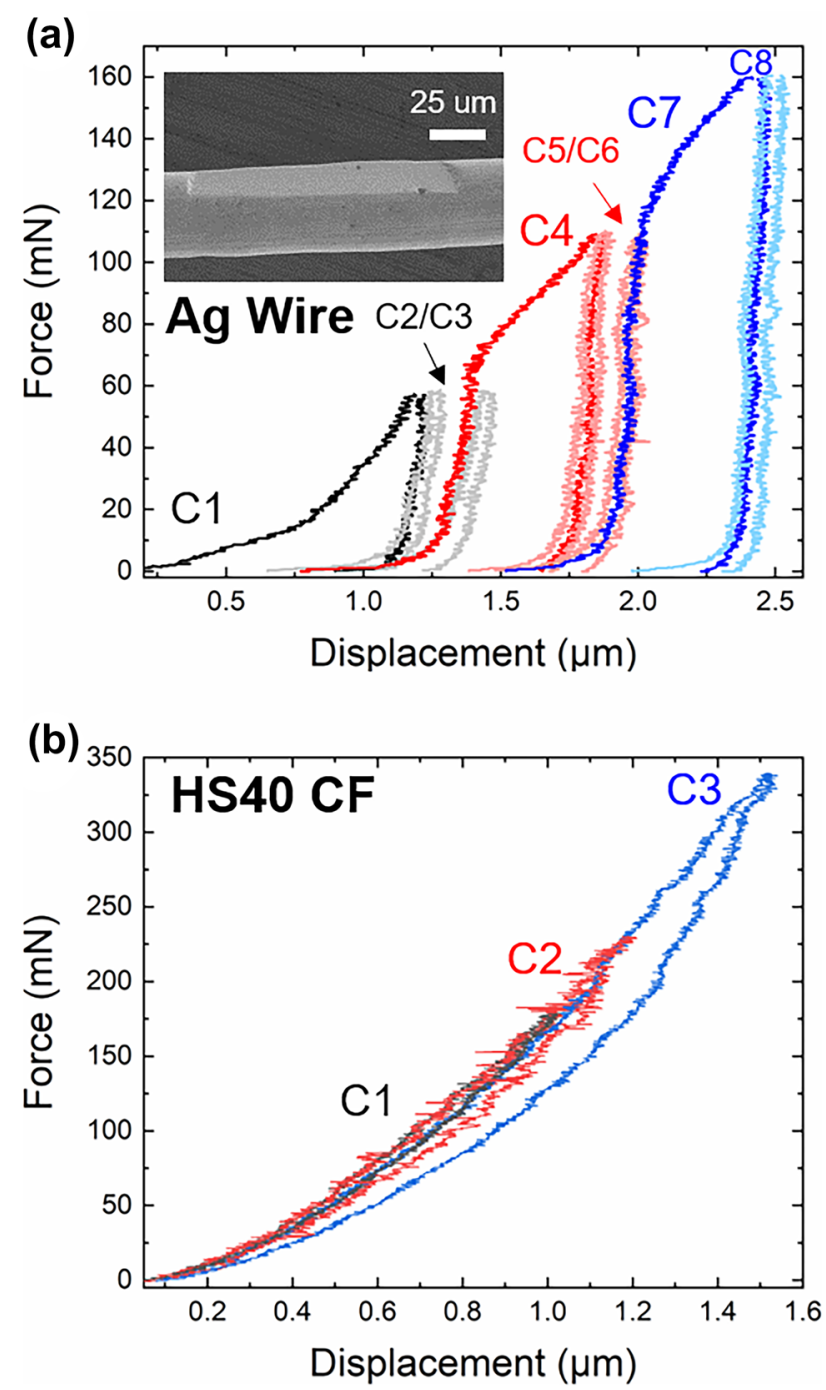

Fig. 4 The load/displacement curves from cyclic compression with progressively increasing loads on (a) Ag wire displaying plasticity and hysteretic behaviour and (b) HS40 CF displaying solely hysteretic behaviour. (inset) SEM image of Ag wire after compression, showing a residual impression

using these hysteretic curves to accurately measure $E_{T}$, load/ unload curves from cyclic compression of HS40 CF (offset in displacement for clarity) are shown in Fig. 5. At lower loads [cycles 1-6 in (a)], the fibre responds elastically to compression. At slightly higher loads [cycles 7-10 in (a)], the unloading curve no longer follows the loading curve, but the residual depth remains negligible, which is indicative of hysteresis. At the highest loads [cycle 11 in (a)], the residual depth is non-zero, which suggests plasticity has occurred. Interestingly, subsequent compression after plasticity [cycle 12 in (a)] to the same load resulted in hysteretic behaviour rather than plasticity. The inset shows the $E_{T}$ calculated using the loading segment of each cycle. There does not appear to be a change in $E_{T}$ as the sample shifts from elastic to hysteretic curves. However, there is an increase in $E_{T}$ at the onset of plasticity, with this increase present in subsequent cycles also despite those cycles displaying hysteretic behaviour. This is similar to the CSS behaviour observed in the Ag wire, where the sample exists in a stable hysteretic loop until sufficient force is applied to induce plasticity, after which the sample enters into a hysteretic loop that gives a higher $E_{T}$ measurement. One key difference for $\mathrm{CF}$ is that no initial plasticity is required before entering into the initial hysteretic loop. One possibility is that $\mathrm{E}_{\mathrm{T}}$ is dependent on the strain rate, similar to the tensile strength of $\mathrm{CF} /$ metal composites being strain-rate dependent [31]. That is, the unloading curve has a different $\mathrm{E}_{\mathrm{T}}$ due to the negative strain rate. It also follows that there would be a different $\mathrm{E}_{\mathrm{T}}$ during the $30 \mathrm{~s}$ between the loading and unloading, where displacement is kept constant (i.e. strain rate $=0$ ), resulting in a stress relaxation that would present itself as an exponential decay of the measured load. Figure 5(b) plots the measured load over time for the cyclic compression presented in Fig. 5(a). There is negligible change in load during the holding period in any of the curves, elastic or otherwise, suggesting $\mathrm{E}_{\mathrm{T}}$ is unlikely to be strain-dependent for HS40 CF. Rather, the hysteresis observed in single crystal materials is due to the presence/movement of dislocations [32], with the shape and stability of the loop dependent on the type and activity of the dislocations [33, 34]. These dislocations are formed during plastic deformation. Hysteretic behaviour (often denoted as anelasticity, reversible plasticity, or viscoelasticity) has previously been observed in a C-based poly-crystalline glassy carbon [35], with the shape of the hysteresis dependent on the crystal structure [36]. CFs are intrinsically polycrystalline, with dislocations pre-existing within its lamellar crystal structure that are likely to contribute to hysteresis [3]. A similar behaviour was also observed in K13312 and TR50S $\mathrm{CF}$ to a lesser extent but was not observed in T300 CF. We propose this hysteretic behaviour is not an intrinsic property of CF. Rather, the extent to which a CF presents hysteretic behaviour is dependent on the presence of pre-existing dislocations in the CF's microstructure, which is due to differing precursors and processing conditions. Therefore, the $E_{T}$ derived from the initial hysteretic cycles before the onset of plasticity can be attributed to the pristine $\mathrm{CF}$ and is an accurate representation of its mechanical properties.

Figure 5(c) shows cyclic compression within the higher load range. The initial compression [cycle 1 in (c)] is hysteretic, with negligible residual depth. However, the second compression [cycle 2 in (c)] has clear residual depth and has plastically deformed, despite having the same maximum load. Subsequent compressions revert to having negligible residual depth, displaying hysteretic behaviour. This suggests that, once above the critical threshold, the onset of 
Fig. 5 The load/displacement curves from cyclic compression of HS40 CF, offset for clarity. (a) 12 cycle compression, with load progressively increasing from 80 to $500 \mathrm{mN}$. Cycle $1-6$ are elastic, cycle $7-10$ exhibit hysteretic behaviour, while plasticity (non-zero residual depth, as highlighted by the circle) occurs in cycle 11 . The force vs time plot for these cyclic compressions is presented in (b). Cycles $1 / 2 / 8$ are held for $20 \mathrm{~s}$, cycles $5 / 6$ are held for $30 \mathrm{~s}$, and the remaining cycles are held for $40 \mathrm{~s}$. (c) 8 cycle compression, with load progressively increasing from 330 to $500 \mathrm{mN}$. Plasticity occurs in cycle 2, with all other cycles exhibiting hysteretic behaviour. (Insets) the $E_{T}$ for each cycle derived from the Morris section

plasticity in $\mathrm{CF}$ is stochastic in nature and systematic compression to a 'safe' maximum load may not be reliable.

While only the loading segment is required to derive $E_{T}$ using the Morris model [26], it is important to also obtain the unloading curve so that the onset of plasticity can be observed from the presence of residual depth in order to avoid deriving an incorrect value for $E_{T}$. Looking at the curves in Fig. 5, the cycles in which plasticity occurs [cycle 11 in (a); cycle2 in (b)] are visually distinct from the hysteretic cycles before and after plasticity. While the difference is most prominent in the non-zero residual depth, the area between the loading and unloading curve for the plastic cycle is also noticeably greater than for the hysteretic cycle. Due to the change in $E_{T}$ after plasticity, it is important to be able to accurately differentiate between plasticity and hysteresis. However, the change in $E_{T}$ after plasticity may vary between samples and may not be as distinct as was observed in the HS40 CF. Indentation studies have previously proposed the consideration of energy-based metrics for the analysis of elasto-plastic deformations [37], which have been applied to hysteretic materials $[36,38]$ via the defining of a ductility index $(D)$ such that:

$D=\frac{A_{L}-A_{U}}{A_{L}}=\frac{A}{A+B}$

where $A_{L}$ and $A_{U}$ are the areas under the loading and unloading curve, respectively and $A$ and $B$ are the areas identified in the inset of Fig. 6. Figure 6 plots $D$ for the cycles presented in Fig. 5 against the maximum load. An approximate linear trend is observed for the hysteretic cycles, with $D$ increasing with maximum load. However, the plastic cycles appear to be outliers. The elastic data points have been excluded from the trend as they should have a ductility index of close to zero by definition. The slight ductility calculated for these elastic cycles are most likely due to noise in the measurement amplified by the division of a very small area within a large area (see Fig. 5a, cycle 3 as an example). Thus, we suggest that the use of ductility index may be useful for discerning if plasticity has occurred in samples that do not have as distinct an increase in $E_{T}$ after plasticity.

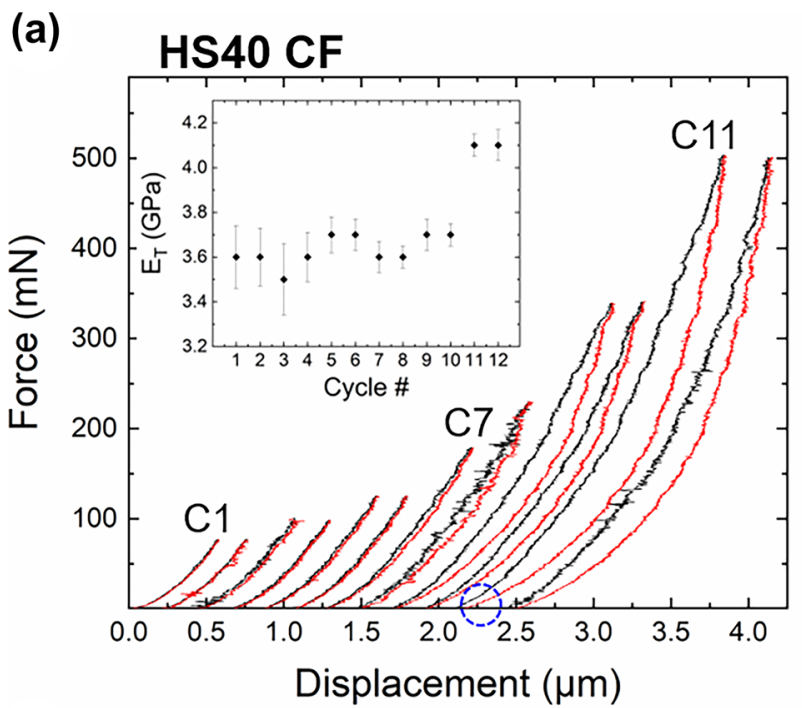

(b)

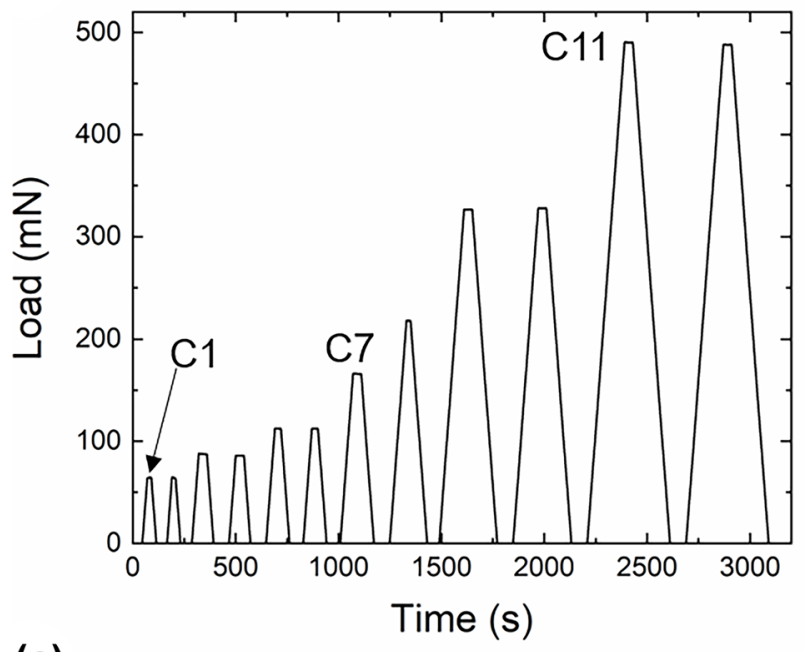

(c)

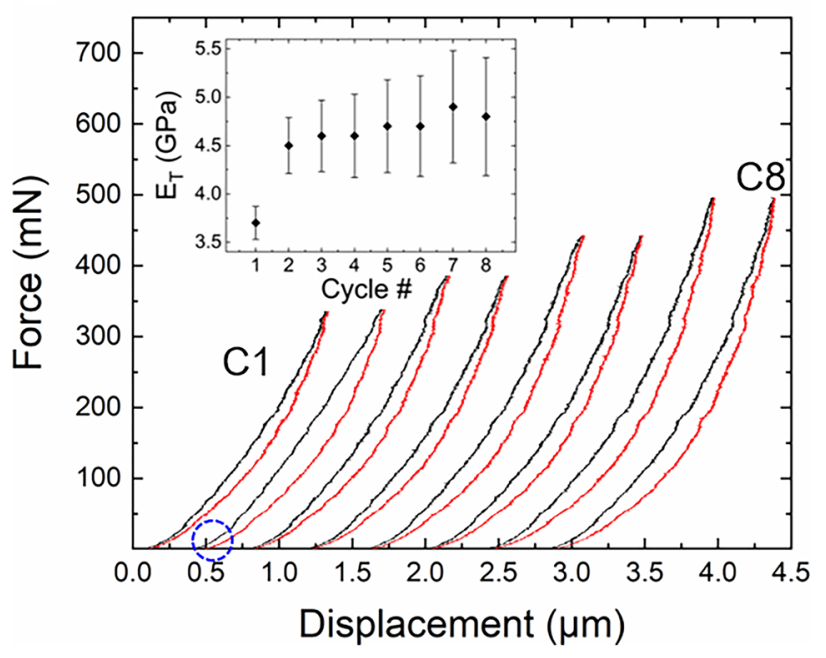




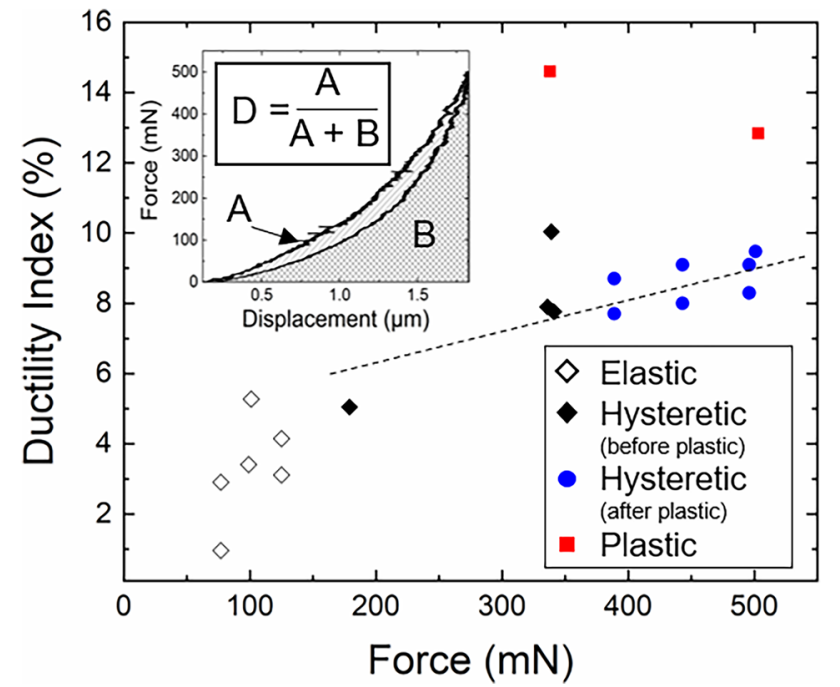

Fig. 6 Ductility index plotted against maximum load. The data points from cycles before plasticity, after plasticity, or from the plastic cycle are diamonds, circles, and squares, respectively. The dotted line through the hysteretic points is a guide for the eyes only. (inset) A curve showing the Ductility Index (D) defined as the area between the loading/unloading curve normalized to the total area under the curve

\section{Discussion}

Figure 7 shows typical load/displacement curves from the different CFs compressed in this study. The $E_{T}$ values were derived by fitting the Morris model (Eq. 1) to the Morris section determined using the method described above for the glass fibre. Figure 8 shows the relationship between $\mathrm{E}_{\mathrm{T}}$ and $\mathrm{E}_{\mathrm{L}}$ for the CFs measured in this study, with the expected inversely proportional relationship [7, 24]. The fitted values for $E_{T}$ are listed in Table 2 alongside the reported values for T300 and values for fibres of a comparable type that were measured using a system equipped with a capacitor external to the region where the compression is occurring, and a correction was applied by estimating the compliance. That study used the elastic contact mechanics model developed by Ward et al. [39], which differs from the Morris by modelling the top load as distributed load rather than a point source. Thus, the Morris section of the curves were also fitted using the Ward model for ease of comparison. (The differences between the two models are covered by Hillbrick et al. [6].) The $\mathrm{E}_{\mathrm{T}}$ for fibres measured using the compression device presented here are consistent with those reported by Naito et al. for fibres of similar type. Interestingly, the standard deviation reported here is significantly lower. We propose that this is due to the compression of intact fibres, rather than cut fibre sections, resulting in greater uniformity in fibre length and the absence of error caused by the ends of the fibre being unconstrained.
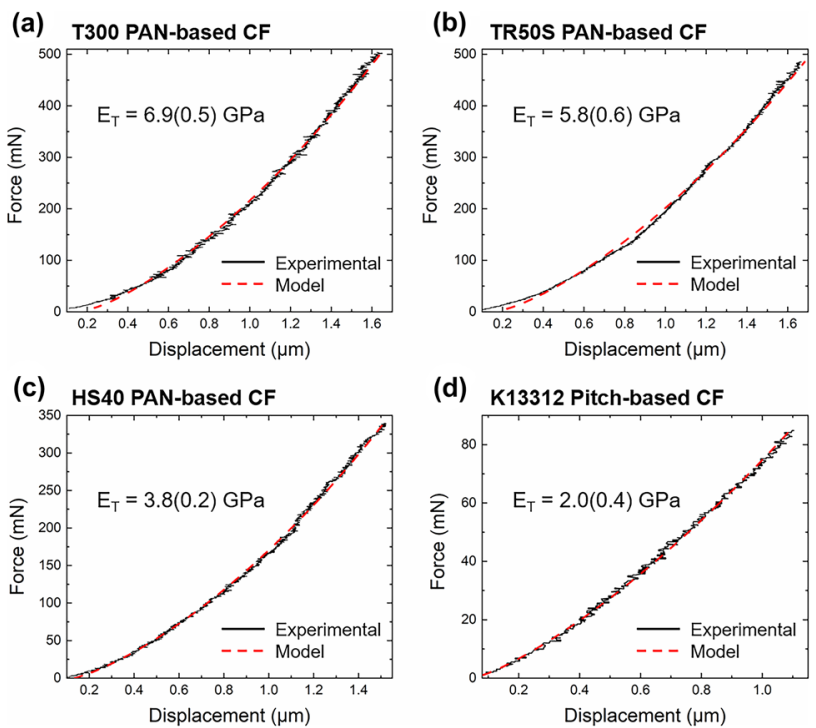

(d) K13312 Pitch-based CF

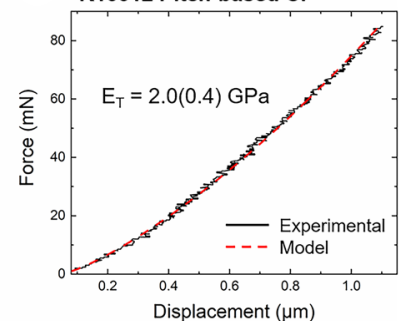

Fig. 7 Typical load/displacement curves of (a) T300, (b) TR50S, (c) HS40, and (d) K13312 CFs fitted using Eq. 1. The average $E_{T}$ derived from the fit across all compressions is indicated on each plot. Numbers given in parentheses are standard deviations

The Morris model, derived from elastic contact mechanics, has been applied in this study to CFs that have behaved purely elastically (i.e. T300), as well as CFs that had curves beyond the elastic regime, from the hysteretic regime to the plastic regime (i.e. HS40). Here, we present a framework for fitting the Morris model to CF curves beyond the elastic regime by utilising the emergence of stable hysteretic loops. During cyclic compression, hysteretic curves are observed before plasticity has occurred. The $\mathrm{E}_{\mathrm{T}}$ fitted from these hysteretic curves agree with those fitted to purely elastic curves,

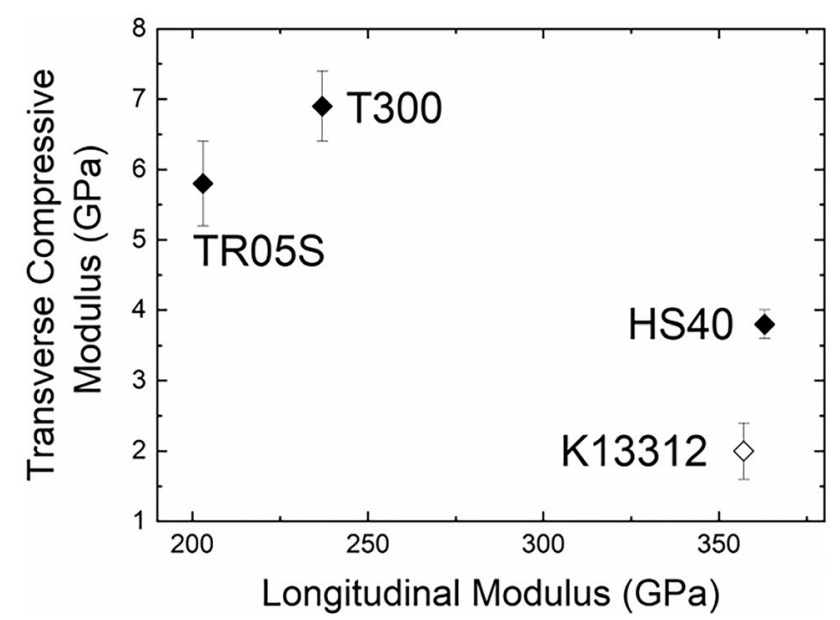

Fig. $8 E_{T}$ plotted against $E_{L}$, showing an inversely proportional relationship. PAN-based CFs are indicated with a solid symbol, while the pitch-based CF is indicated with an empty symbol 
Table 2 The $\mathrm{E}_{\mathrm{T}}$ values measured in the current study using the model developed by Morris et al. and Ward et al. These are compared to the values reported by Ref. [24], which uses the model developed by Ward et al. for different, but similar fibre types (apart from T300). Numbers given in parenthesis are standard deviations

\begin{tabular}{lllll}
\hline Fibre & \multicolumn{2}{l}{ This Study (GPa) } & Fibre Type & $\begin{array}{l}\text { Naito et al. } \\
\text { [24] (GPa) }\end{array}$ \\
\cline { 2 - 4 } & Morris Model & Ward Model & & \\
\hline T300 & $6.9(0.5)$ & $8.2(0.8)$ & T300 & $7.8(2.9)$ \\
HS40 & $5.8(0.6)$ & $6.5(0.7)$ & $\begin{array}{l}\text { High Strength } \\
\text { PAN }\end{array}$ & $6-10$ \\
TR50S & $3.8(0.2)$ & $4.3(0.3)$ & $\begin{array}{c}\text { High Modulus } \\
\text { PAN }\end{array}$ & $3-6$ \\
K13312 & $2.0(0.4)$ & $2.1(0.4)$ & K-series Pitch & $1.5-2$ \\
\hline
\end{tabular}

indicating that the Morris model provides an accurate and reproducible description for curves within a stable hysteretic loop as this hysteresis is due to reversible movement of dislocations that were already present within the pristine fibre. Once plasticity occurs, the fibre enters a different stable hysteretic loop. In practice, the slight plasticity has transformed the initial CF into a new material that may have a slightly compressed cross-section or an increased number of dislocations. Results from T300 and TR50S CF in this work (as well as others in the literature) show that the Morris model can be reasonably applied to a CF with a slightly non-circular cross-section. Further, the presence of dislocations causes hysteresis but does not affect the fitted $\mathrm{E}_{\mathrm{T}}$. Thus, when the Morris model was applied to curves within this new stable hysteretic loop, a consistent $\mathrm{E}_{\mathrm{T}}$ was fitted for all curves within this loop. This new $\mathrm{E}_{\mathrm{T}}$ is representative of the slightly modified CF that was created by the slight plasticity. We believe this process of slight plasticity into a new stable hysteretic loop can occur multiple times with the same $\mathrm{CF}$, similar to that observed in the $\mathrm{Ag}$ wire, until a critical strain is reached such that breakage occurs.

Having developed a suitable technique to measure $\mathrm{E}_{\mathrm{T}}$ our focus will shift to identifying processing conditions for polyacrylonitrile fibre that led to microstructural changes in precursor fibre and the final $\mathrm{CF}$ that would enable the production of CFs with an industrially useful $E_{T}: E_{L}$ ratio.

\section{Conclusion}

A compression device that directly measures the mechanical strain of fibres during transverse compression testing using low coherence speckle tracking has been presented. This device overcomes the limitation caused by the difficulty in measuring compliance near the resolution limit by directly measuring the displacement. Indeed, only $<0.5 \mathrm{~mm}$ of diamond and tungsten are present between the sample and the point of measurement.
The accuracy of the device was confirmed by compression of glass fibre, an isotropic material, along the transverse direction. The experimental data was fitted using the model for transverse compression developed by Morris et al. and a transverse compressive modulus of $E_{T}=82(9)$ $\mathrm{GPa}$ was measured. This is consistent with the glass fibre's longitudinal modulus $E_{L}=86.3(2.3) \mathrm{GPa}$.

Several carbon fibres (CF) were also tested and found to be consistent with the literature values of fibres of similar type. Ductile silver filament wire was characterized to clearly illustrate known cyclic stress strain behaviours of materials. These observations were used to aid the interpretation of results obtained from CF.

Further, three distinct regions within the compression's load/displacement curves were identified, an initial section representing fibre alignment between the diamond tips, a section where the curve was well-fit by the model, and a non-elastic section after the onset of plasticity. A method using the ductility index was proposed in order to identify the onset of plasticity, especially for fibres which display hysteretic properties.

Acknowledgements The authors would like to acknowledge Julius Orwa for providing the carbon fibres for this study, Nicole Phair-Sorenson, Lucille Cotter, Colin Veitch, and Debra Hamilton for performing the Favimat and SEM results, and the CSIRO Waurn Ponds mechanical workshop staff for the construction of components used in the fibre compression test system.

Funding This research did not receive any specific grant from funding agencies in the public, commercial, or not-for-profit sectors.

\section{Declarations}

Conflicts of Interest The authors declare that they have no known competing financial interests or personal relationships that could have appeared to influence the work reported in this paper.

Open Access This article is licensed under a Creative Commons Attribution 4.0 International License, which permits use, sharing, adaptation, distribution and reproduction in any medium or format, as long as you give appropriate credit to the original author(s) and the source, provide a link to the Creative Commons licence, and indicate if changes were made. The images or other third party material in this article are included in the article's Creative Commons licence, unless indicated otherwise in a credit line to the material. If material is not included in the article's Creative Commons licence and your intended use is not permitted by statutory regulation or exceeds the permitted use, you will need to obtain permission directly from the copyright holder. To view a copy of this licence, visit http://creativecommons.org/licenses/by/4.0/.

\section{References}

1. Fitzer E (1989) Pan-based carbon fibers-present state and trend of the technology from the viewpoint of possibilities and limits to influence and to control the fiber properties by the process parameters. Carbon 27(5):621-645 
2. Krenkel W, Berndt F (2005) C/C-SiC composites for space applications and advanced friction systems. Mat Sci Eng a-Struct 412(1-2):177-181. https://doi.org/10.1016/j.msea.2005.08.204

3. Newcomb BA (2016) Processing, structure, and properties of carbon fibers. Compos Part A-Appl Sci Manufact 91:262-282. https://doi.org/10.1016/j.compositesa.2016.10.018

4. Edie DD (1998) The effect of processing on the structure and properties of carbon fibers. Carbon 36(4):345-362. https://doi. org/10.1016/S0008-6223(97)00185-1

5. Paris O, Peterlik H (2009) The structure of carbon fibres. Woodhead Publ Text 88b:353-377. https://doi.org/10.1533/9781845697310.3. 353

6. Hillbrick LK, Kaiser J, Huson MG, Naylor GRS, Wise ES, Miller AD, Lucas S (2019) Determination of the transverse modulus of cylindrical samples by compression between two parallel flat plates. Sn Appl Sci 1(7):724-738. https://doi.org/10.1007/ s42452-019-0726-7

7. Kawabata S (1990) Measurement of the transverse mechanicalproperties of high-performance fibers. J Text Inst 81(4):432-447. https://doi.org/10.1080/00405009008658721

8. Cheng M, Chen W, Weerasooriya T (2004) Experimental investigation of the transverse mechanical properties of a single Kevlar ${ }^{\circledR}$ KM2 fiber. Int J Solids Struct 41(22-23):6215-6232

9. Oya N, Johnson DJ (2001) Longitudinal compressive behaviour and microstructure of PAN-based carbon fibres. Carbon 39(5):635-645. https://doi.org/10.1016/S0008-6223(00)00147-0

10. Kumar IP, Mohite P, Kamle S (2013) Axial compressive strength testing of single carbon fibres. Arch Mech 65(1):27-43

11. Csanádi T, Németh D, Zhang C, Dusza J (2017) Nanoindentation derived elastic constants of carbon fibres and their nanostructural based predictions. Carbon 119:314-325

12. Dyer S, Lord D, Hutchinson I, Ward I, Duckett R (1992) Elastic anisotropy in unidirectional fibre reinforced composites. J Phys D Appl Phys 25(1):66-76

13. LLorca J, González C, Molina-Aldareguía JM, Segurado J, Seltzer R, Sket F, Rodríguez M, Sádaba S, Muñoz R, Canal LP (2011) Multiscale modeling of composite materials: a roadmap towards virtual testing. Adv Mater 23(44):5130-5147

14. Herráez M, Mora D, Naya F, Lopes CS, González C, LLorca J, (2015) Transverse cracking of cross-ply laminates: A computational micromechanics perspective. Compos Sci Technol 110:196-204

15. Malekimoghadam R, Icardi U (2019) Prediction of mechanical properties of carbon nanotube-carbon fiber reinforced hybrid composites using multi-scale finite element modelling. Compos Part B: Engineer 177:107405 (1-15)

16. Miyagawa H, Mase T, Sato C, Drown E, Drzal LT, Ikegami K (2006) Comparison of experimental and theoretical transverse elastic modulus of carbon fibers. Carbon 44(10):2002-2008

17. Ishikawa T, Koyama K, Kobayashi S (1977) Elastic moduli of carbon-epoxy composites and carbon fibers. J Compos Mater 11(3):332-344

18. Sugimoto Y, Kato T, Shioya M, Kobayashi T, Sumiya K, Fujie M (2013) Structure change of carbon fibers during axial compression. Carbon 57:416-424. https://doi.org/10.1016/j.carbon.2013. 02.014

19. Nakatani M, Shioya M, Yamashita J (1999) Axial compressive fracture of carbon fibers. Carbon 37(4):601-608

20. Oya N, Johnson DJ (1999) Direct measurement of longitudinal compressive strength in carbon fibres. Carbon 37(10):1539-1544

21. Guo Z, Casem D, Hudspeth M, Nie X, Sun J, Chen W (2016) Transverse compression of two high-performance ballistic fibers. Text Res J 86(5):502-511
22. Maurin R, Davies P, Baral N, Baley C (2008) Transverse properties of carbon fibres by nano-indentation and micro-mechanics. Appl Compos Mater 15(2):61-73

23. Duan S, Liu F, Pettersson T, Creighton C, Asp LE (2020) Determination of transverse and shear moduli of single carbon fibres. Carbon 158:772-782

24. Naito K, Tanaka Y, Yang J-M (2017) Transverse compressive properties of polyacrylonitrile (PAN)-based and pitch-based single carbon fibers. Carbon 118:168-183. https://doi.org/10.1016/j. carbon.2017.03.031

25. Guo Z, Chen W, Zheng J (2019) Improved quasi-static twin-fiber transverse compression of several high-performance fibers. Text Res J 89(9):1595-1613. https://doi.org/10.1177/0040517518775927

26. Morris S (1968) The Determination of the Lateral-Compression Modulus of Fibres. J Text Inst 59(11):536-547

27. Smith DR, Fickett F (1995) Low-temperature properties of silver. J Res Nat Inst Stand Technol 100(2):119-171

28. Hales R, Holdsworth S, O'Donnell M, Perrin I, Skelton R (2002) A code of practice for the determination of cyclic stress-strain data. Mater High Temp 19(4):165-185

29. Jiang Y, Zhang J (2008) Benchmark experiments and characteristic cyclic plasticity deformation. Int J Plast 24(9):1481-1515

30. Skelton R, Maier H, Christ H-J (1997) The Bauschinger effect, Masing model and the Ramberg-Osgood relation for cyclic deformation in metals. Mater Sci Eng, A 238(2):377-390

31. Zhou Y, Wang Y, Jeelani S, Xia Y (2007) Experimental study on tensile behavior of carbon fiber and carbon fiber reinforced aluminum at different strain rate. Appl Compos Mater 14(1):17-31

32. Sivaprasad S, Paul SK, Das A, Narasaiah N, Tarafder S (2010) Cyclic plastic behaviour of primary heat transport piping materials: Influence of loading schemes on hysteresis loop. Mater Sci Eng, A 527(26):6858-6869

33. Mayama T, Sasaki K, Kuroda M (2008) Quantitative evaluations for strain amplitude dependent organization of dislocation structures due to cyclic plasticity in austenitic stainless steel $316 \mathrm{~L}$. Acta Mater 56(12):2735-2743

34. Gaudin C, Feaugas X (2004) Cyclic creep process in AISI $316 \mathrm{~L}$ stainless steel in terms of dislocation patterns and internal stresses. Acta Mater 52(10):3097-3110

35. Fielda J, Swain M (1996) The indentation characterisation of the mechanical properties of various carbon materials: Glassy carbon, coke and pyrolytic graphite. Carbon 34(11):1357-1366

36. Iwashita N, Field J, Swain M (2002) Indentation hysteresis of glassy carbon materials. Philos Mag A 82(10):1873-1881

37. Sakai M, Shimizu S, Ishikawa T (1999) The indentation loaddepth curve of ceramics. J Mater Res 14(4):1471-1484

38. Huang X, Shiell T, de Tomas C, Suarez-Martinez I, Wong S, Mann S, McKenzie D, Marks N, McCulloch D, Bradby J (2020) The mechanical response of glassy carbon recovered from high pressure. Journal of Applied Physics 127 (14):145105 (1-7)

39. Jawad SA, Ward I (1978) The transverse compression of oriented nylon and polyethylene extrudates. J Mater Sci 13(7):1381-1387

Publisher's Note Springer Nature remains neutral with regard to jurisdictional claims in published maps and institutional affiliations. 